The Astrophysical Journal, 482:230-244, 1997 June 10

(C) 1997. The American Astronomical Society. All rights reserved. Printed in U.S.A.

\title{
THE MHD KELVIN-HELMHOLTZ INSTABILITY. II. THE ROLES OF WEAK AND OBLIQUE FIELDS IN PLANAR FLOWS
}

\author{
T. W. JoNes, ${ }^{1,2,3}$ JoSEPH B. GaAlaAs, ${ }^{1,2,4}$ Dongsu RyU,,${ }^{5,6,7}$ AND AdAM FranK ${ }^{1,8,9}$ \\ Received 1996 November 13; accepted 1997 January 8
}

\begin{abstract}
We have carried out high-resolution MHD simulations of the nonlinear evolution of KelvinHelmholtz unstable flows in $2 \frac{1}{2}$ dimensions. The modeled flows and fields were initially uniform except for a thin shear layer with a hyperbolic tangent velocity profile and a small, normal mode perturbation. These simulations extend work by Frank et al. and Malagoli, Bodo, \& Rosner. They consider periodic sections of flows containing magnetic fields parallel to the shear layer, but projecting over a full range of angles with respect to the flow vectors. They are intended as preparation for fully three-dimensional calculations and to address two specific questions raised in earlier work: (1) What role, if any, does the orientation of the field play in nonlinear evolution of the MHD Kelvin-Helmholtz instability in $2 \frac{1}{2}$ dimensions? (2) Given that the field is too weak to stabilize against a linear perturbation of the flow, how does the nonlinear evolution of the instability depend on strength of the field? The magnetic field component in the third direction contributes only through minor pressure contributions, so the flows are essentially two-dimensional. In Frank et al. we found that fields too weak to stabilize a linear perturbation may still be able to alter fundamentally the flow so that it evolves from the classical "Cat's Eye" vortex expected in gasdynamics into a marginally stable, broad laminar shear layer. In that process the magnetic field plays the role of a catalyst, briefly storing energy and then returning it to the plasma during reconnection events that lead to dynamical alignment between magnetic field and flow vectors. In our new work we identify another transformation in the flow evolution for fields below a critical strength. That we found to be $\sim 10 \%$ of the critical field needed for linear stabilization in the cases we studied. In this "very weak field" regime, the role of the magnetic field is to enhance the rate of energy dissipation within and around the Cat's Eye vortex, not to disrupt it. The presence of even a very weak field can add substantially to the rate at which flow kinetic energy is dissipated.

In all of the cases we studied magnetic field amplification by stretching in the vortex is limited by tearing mode, "fast" reconnection events that isolate and then destroy magnetic flux islands within the vortex and relax the fields outside the vortex. If the magnetic tension developed prior to reconnection is comparable to Reynolds stresses in the flow, that flow is reorganized during reconnection. Otherwise, the primary influence on the plasma is generation of entropy. The effective expulsion of flux from the vortex is very similar to that shown by Weiss for passive fields in idealized vortices with large magnetic Reynolds numbers. We demonstrated that this expulsion cannot be interpreted as a direct consequence of steady, resistive diffusion, but must be seen as a consequence of unsteady fast reconnection.
\end{abstract}

Subject headings: instabilities - MHD — plasmas — turbulence

\section{INTRODUCTION}

Weak magnetic fields threading conducting fluid media can play vital dynamical roles, even when traditional criteria, such as relative magnetic and gas pressures, suggest the fields are entirely negligible. Perhaps the best example of this is the destabilizing influence of a vanishingly small magnetic field crossing a Keplerian accretion disk (Balbus \& Hawley 1991), where the mere presence of the field seems fundamentally to alter the local flow properties. Other examples abound, however, that could be particularly important in astrophysics. Among them, we would include

\footnotetext{
${ }^{1}$ Department of Astronomy, University of Minnesota, Minneapolis, MN 55455.

${ }^{2}$ Minnesota Supercomputer Institute, University of Minnesota, Minneapolis, MN 55455.

3 twj@astro.spa.umn.edu.

${ }^{4}$ gaalaas@msi.umn.edu.

5 Department of Astronomy and Space Science, Chungnam National University, Daejeon 305-764, Korea.

${ }^{6}$ Department of Astronomy, University of Washington, Box 351580, Seattle, WA 98195-1580.

7 ryu@sirius.chungnam.ac.kr.

${ }^{8}$ Department of Physics and Astronomy, University of Rochester, Rochester, NY 14627-0171.

9 afrank@alethea.pas.rochester.edu.
}

weak fields penetrating turbulent or otherwise strongly unstable flows, where such fields significantly alter evolution and transport properties (e.g., Biskamp \& Welter 1989; Cattaneo \& Vainstein 1991; Nordlund et al. 1992; Jun, Norman, \& Stone 1995). Sheared motion is a critical common element in many of these flows, and the consequent stretching of a weak, but a large-scale field can lead to a locally enhanced role for the field. The flows will also frequently lead to current sheets and associated magnetic field topologies unstable to reconnection, and that is central to the nonlinear evolution of the systems (e.g., Biskamp 1993; Parker 1994). Through these processes the fields can also have more global consequences. Study of the nonlinear evolution of the classical Kelvin-Helmholtz $(\mathrm{KH})$ instability could be particularly useful as a well-defined example of strongly sheared flows. Further, since KH unstable boundary layers are probably common, the behavior of the instability is important for its own sake.

Although the $\mathrm{KH}$ instability is fairly well studied in ordinary hydrodynamics (e.g., Corcos \& Sherman 1984), comparable study has been much slower in magnetohydrodynamics (MHD). That is because the magnetic field substantially complicates the physics itself and also because 
computational methods and resources needed for such studies are only recently up to the task. The linear analysis of the MHD KH instability is relatively straightforward and was long ago carried out for a number of simple flow and field configurations (e.g., Chandrasekhar 1961; Miura \& Pritchett 1982). Generally, and especially if the velocity change is not supersonic, the ordinary fluid shear layer is unstable to perturbations with wavevectors in the plane of the shear layer and with wavelengths greater than the thickness of the layer (e.g., Miura 1990). When there is a field component projecting onto the flow field, magnetic tension provides a stabilizing influence. A simple vortex sheet is stabilized against linear perturbations whenever the magnetic field strength is sufficient that $c_{\mathrm{A}}>\left|\left(\hat{\boldsymbol{k}} \cdot \boldsymbol{U}_{0}\right)\right|$ $\left(2 \hat{\boldsymbol{k}} \cdot \hat{\boldsymbol{B}}_{0}\right) \mid$, where $\boldsymbol{U}_{0}$ is the velocity difference between the two layers, $c_{\mathrm{A}}$ is the Alfvén speed, $\boldsymbol{k}$ is the perturbation wavevector, and $\hat{\boldsymbol{B}}_{0}$ is the direction of the magnetic field (Chandrasekhar 1961).

Frank et al. (1996, hereafter Paper I) and Malagoli, Bodo, \& Rosner (1996, hereafter MBR) recently presented complementary nonlinear analyses of the MHD KH instability in mildly compressible flows based on two-dimensional numerical simulations carried out with new (and different) Riemann-solver-based MHD codes. While not the first numerical studies of the MHD KH instability, they represented big improvements over previous calculations in both numerical resolution and extent to which flow evolution was followed toward asymptotic states (readers are referred to Paper I for additional, earlier citations). Considering perturbed two-dimensional flows that were uniform except for a thin, smooth velocity transition layer, those two papers emphasized the qualitatively different behaviors in the nonlinear evolution of unstable flows depending on how close the field strength is to its critical strength for stabilization. For fields only slightly below the critical value, enhancements in the tension of the field through linear growth can stabilize the flow before it develops distinctly nonlinear characteristics. For weaker fields, however, the initial evolution of the instability is very similar to that for the ordinary $\mathrm{KH}$ instability. That results in the formation of eddies, and hence, to substantial stretching of the magnetic field lines as well as reconnection. Paper I emphasized the remarkable fact that in a case with a field 2.5 times weaker than critical, reconnection can lead to selforganization in the flow and fairly rapid relaxation to a quasi-steady laminar and marginally stable flow. MBR presented summaries of simulations extending to somewhat weaker fields showing evidence for similar behaviors.

Neither Paper I nor MBR, however, explored the problem in sufficient depth to establish the conditions necessary for the previously mentioned self-organization. In addition, it is very important in this situation to understand how the magnetic fields behave when they are "very" weak (a concept whose definition needs clarification, in fact). A closely related matter is what differences, if any, exist between the behavior of a truly weak field and a stronger field whose projection onto the flow vectors is weak. Alternatively stated, are there differences between the nonlinear two-dimensional MHD KH instability and the " $2 \frac{1}{2}$ dimensional" MHD KH instability? Answers to those basic questions are the objective of this paper. We find: (1) for the cases we have considered with an initially uniform field that the magnetic field transverse to the plane is unimportant and (2) there is a transition from the role of the magnetic field as a catalyst to flow self-organization to a role as an added source of energy dissipation that should vanish directly as the initial magnetic field strength projected onto the plane vanishes. Ultimately, we must understand the full three-dimensional problem, in which the perturbation wavevector also lies outside the flow direction. On the other hand, it has been difficult to carry out threedimensional MHD simulations with sufficient numerical resolution to be confident of the results in complex flows such as these. In addition, it will be useful to compare fully three-dimensional behaviors with two-dimensional flows. We hope that the current work is a significant, constructive step toward a full understanding of this problem.

The paper plan is as follows. In $\S 2$ we will summarize the problem setup and relevant results from Paper I. Section 3 contains a discussion of new results, while $\S 4$ provides a brief summary and conclusion. We also include an Appendix presenting an analytical model for diffusive flux expulsion from a steady vortex, in order to contrast that physics with what we observe in the eddies that form in our simulations.

\section{BACKGROUND}

In order to focus on specific, important physical issues we have chosen to explore an idealization of the MHD KH problem, reserving for the future the more general problem. We present in this section only a bare outline of our method and some key results from previous work. A full discussion of the computational setup along with several tests of such issues as adequate numerical resolution and geometry of the computational box can be found in Paper I.

\subsection{Problem Definition}

The geometry of the computations is shown in Figure 1. The only difference from Paper $I$ is that in those earlier computations we assumed an aligned field, $\theta=\cos ^{-1}$ $\left|\hat{\boldsymbol{B}}_{0} \cdot \hat{\boldsymbol{U}}_{0}\right|=0$, whereas we now relax that constraint to include magnetic fields oblique or orthogonal to the flow plane. We assume the flow to be periodic in $x$ and that the $y$ boundaries are reflecting (i.e., neither flow nor field lines cross the $y$ boundaries). This was a configuration used initially by Miura (1984), and we followed it in Paper I to enable a direct comparison with his results. The influences of those boundary choices are discussed fully in Paper I. In brief, the periodic boundary limits coalescence of structures to scales equal to the box dimension, $L$. That is more significant than the existence of the reflecting boundaries, which seem to have only minor influence on dynamics in the (narrow) central regions where flow organization and dissipation is largely determined.

The initial background flow has uniform density, $\rho=1$, gas pressure, $p=0.6$, and an adiabatic index, $\gamma=5 / 3$, so that the sound speed, $c_{s}=(\gamma p / \rho)^{1 / 2}=1.0$. The magnetic field, $\boldsymbol{B}_{0}=\boldsymbol{B}_{0}(\hat{\boldsymbol{x}} \cos \theta+\hat{\boldsymbol{z}} \sin \theta)$, is also uniform. In Paper I we considered cases with $B_{0}=0.4,0.2$ (and $\theta=0$ ), so that $M_{\mathrm{A}}=2.5,5$, since those were studied by Miura (1984). We now add to these a number of new cases as outlined in Table 1 . To facilitate comparisons we identify the simulations from Paper $I$ as cases 1 and 2 in Table 1, with the new simulations following. The velocity in the background state is antisymmetric about $y=L / 2$ according to the relation

$$
\boldsymbol{u}_{0}=u_{0}(y) \hat{\boldsymbol{x}}=-\frac{U_{0}}{2} \tanh \left(\frac{y-L / 2}{a}\right) \hat{\boldsymbol{x}},
$$



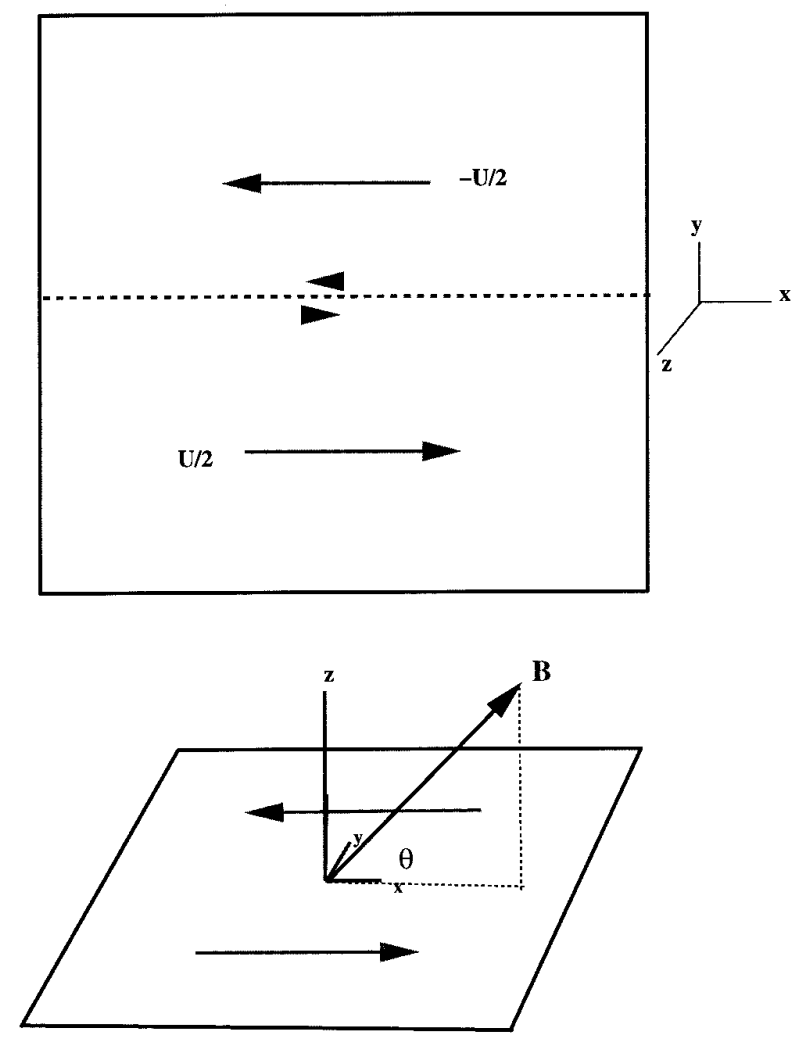

FIG. 1.-Cartoon illustrating the computational setup for these simulations. There is a central shear layer separating uniform flows. Except for the initial perturbation the flow conditions are otherwise uniform. The magnetic field projects onto the computational plane at an angle, $\theta$.

with $U_{0}=1$. This describes a smoothly varying flow within a shear layer of full width $2 a$. For all our simulations presented here $a=L / 25$, chosen to make the interactions with the reflecting boundaries small. The square computational box has $L=2.51$. Flow is to the left in the top half-plane and to the right below that. To this state we add a perturbation, $\delta(\rho, p, \boldsymbol{u}, \boldsymbol{B})$, defined to be a normal mode found from the linearized MHD equations appropriate for the chosen background, periodic in $x$ and evanescent in $y$, with period equal to the length of the computational box, $L$. This was done exactly as in Paper I. We note that when $\theta \neq 0$ there are perturbations in all three vector components of the magnetic and velocity fields. Under the conditions we used the computational frame is comoving with the $\mathrm{KH}$ waves. All flow and field quantities are either symmetric or antisymmetric around two points, which happen with our choice of phases in $\delta(\rho, p, \boldsymbol{u}, \boldsymbol{B})$ to be at $y=L / 2, x=$ $L / 4,3 L / 4$. Because the velocity field is antisymmetric around these points they are the places where strong vortices tend to form.

The equations we solve numerically are those of ideal compressible MHD; namely,

$$
\begin{gathered}
\frac{\partial \rho}{\partial t}+\nabla \cdot(\rho \boldsymbol{u})=0 \\
\frac{\partial \boldsymbol{u}}{\partial t}+\boldsymbol{u} \cdot \nabla \boldsymbol{u}+\frac{1}{\rho} \nabla p-\frac{1}{\rho}(\nabla \times \boldsymbol{B}) \times \boldsymbol{B}=0 \\
\frac{\partial p}{\partial t}+\boldsymbol{u} \cdot \nabla p+\gamma p \nabla \cdot \boldsymbol{u}=0 \\
\frac{\partial \boldsymbol{B}}{\partial t}-\nabla \times(\boldsymbol{u} \times \boldsymbol{B})=0
\end{gathered}
$$

along with the constraint $\boldsymbol{\nabla} \cdot \boldsymbol{B}=0$ imposed to account for the absence of magnetic monopoles (e.g., Priest 1984). The isentropic gas equation of state is $p \propto \rho^{\gamma}$. Standard symbols are used for common quantities. Here, we have chosen rationalized units for the magnetic field so that the magnetic pressure $p_{b}=B^{2} / 2$ and the Alfvén speed is simply $c_{\mathrm{A}}=$ $B / \rho^{1 / 2}$.

These equations were solved using a multidimensional MHD code based on the explicit, finite difference "total variation diminishing" or "TVD" scheme. The method is an MHD extension of the second-order finite-difference, upwinded, conservative gasdynamics scheme of Harten (1983), as described by Ryu \& Jones (1995). The multidimensional version of the code, along with a description of various one and two-dimensional flow tests is contained in Ryu, Jones, \& Frank (1995). The code contains an fft-based routine that maintains the $\nabla \cdot \boldsymbol{B}=0$ condition at each time

TABLE 1

SUMMARY OF MHD KH SimULATIONS

\begin{tabular}{ccccccccc}
\hline \hline Case $^{\mathrm{a}}$ & $B_{0}=c_{\mathrm{A}} / c_{s}{ }^{\mathrm{b}}$ & $\begin{array}{c}\theta \\
(\mathrm{deg})\end{array}$ & $B_{p o}=B_{0} \cos \theta$ & $M_{\mathrm{A}}{ }^{\mathrm{b}}$ & $\beta_{0}^{\mathrm{b}}$ & $t_{g}{ }^{\mathrm{c}}$ & $\begin{array}{c}\text { End } \\
\text { Time }^{\mathrm{c}}\end{array}$ & $N_{x}{ }^{\mathrm{d}}$ \\
\hline $1^{\mathrm{e}} \ldots \ldots$. & 0.4 & $0^{\circ}$ & 0.4 & 2.5 & 7.5 & 3.79 & $20 \tau$ & $512^{\mathrm{e}}$ \\
$2^{\mathrm{e}} \ldots \ldots$. & 0.2 & $0^{\circ}$ & 0.2 & 5.0 & 30 & 1.86 & $20 \tau$ & $512^{\mathrm{e}}$ \\
$3 \ldots \ldots \ldots$ & 0.2 & $45^{\circ}$ & 0.14 & 5.0 & 30 & 1.71 & $30 \tau$ & 512 \\
$4 \ldots \ldots \ldots$ & 0.14 & $0^{\circ}$ & 0.14 & 7.07 & 60 & 1.71 & $30 \tau$ & 512 \\
$5 \ldots \ldots \ldots$ & 0.2 & $90^{\circ}$ & 0.0 & 5.0 & 30 & 1.59 & $20 \tau$ & 256 \\
$6 \mathrm{~h} \ldots \ldots$. & 0.2 & $85^{\circ}$ & 0.02 & 5.0 & 30 & 1.59 & $50 \tau$ & 512 \\
$6 \mathrm{~m} \ldots \ldots$. & 0.2 & $85^{\circ}$ & 0.02 & 5.0 & 30 & 1.59 & $30 \tau$ & 256 \\
$7 \ldots \ldots \ldots$ & 0.02 & $0^{\circ}$ & 0.02 & 50 & 3000 & 1.59 & $30 \tau$ & 256 \\
$8 \ldots \ldots \ldots$ & 0.04 & $0^{\circ}$ & 0.04 & 25 & 750 & 1.67 & $30 \tau$ & 256 \\
$9 \ldots \ldots \ldots$ & 0.07 & $0^{\circ}$ & 0.07 & 14.3 & 245 & 1.67 & $20 \tau$ & 256 \\
\hline
\end{tabular}

${ }^{a}$ All models have used $\gamma=5 / 3, M=U_{0} / c_{s}=1, c_{s}=1, L=2.51$, and $a=L / 25$

b The Alfvén speed, $c_{\mathrm{A}}=B / \rho^{1 / 2}$, Alfvén Mach number, and $\beta_{0}=p_{g} / p_{b}=(2 / \gamma)\left(M_{\mathrm{A}} / M\right)^{2}$ here refer to the total initial magnetic field strength, not just that projected onto the plane of the flow.

${ }^{c}$ The growth time is an approximation to the inverse linear growth rate; namely $t_{g}=1 / \Gamma ; \tau=t / t_{g}$.

${ }^{d}$ Computations were carried out on a square grid of the size indicated, with $N_{y}=N_{x}$. Periodic boundaries were assumed for $x$ and reflecting boundaries were assumed for $y$.

e Cases 1 and 2 were presented and discussed in Paper I. They are cited here for reference. Each was computed with two or more numerical resolutions, with the largest listed here. 
step within machine accuracy. That step does not compromise the other conservation relations.

Numerical solution of equations (2)-(5) on a discrete grid leads, through truncation errors, to diffusion of energy, and momentum, as well as to entropy generation. Of course, such effects are also present in nature and are important to defining the character of the flows. The existence of effective numerical resistivity is necessary, for example, to allow magnetic reconnection to occur in the calculations. Our methods exactly conserve total energy, as well as mass, momentum, and magnetic flux, so the exchange between kinetic, thermal and magnetic energies along with entropy production is internally consistent. There is fairly good evidence that conservative monotonic schemes, as this one is, do a good job of approximately representing physical viscous and resistive dissipative processes that are expected to take place on scales smaller than the grid (e.g., Porter \& Woodward 1994). For the astrophysical environments being simulated the expected dissipative scales are likely very much smaller than those that can be modeled directly. Recent numerical studies of reconnection suggest in the MHD limit with large kinetic and magnetic Reynolds numbers that the local energy dissipation rate through reconnection becomes independent of the value of the resistivity in complex flows (e.g., Biskamp 1993, 1994). However, when we depend on numerical dissipation, we must be cautious about the possible role of "uncaptured" dynamical structures that could be expected on scales smaller than the grid scale, or about magnetic field structures on such scales that could enhance the number of reconnection sites. In fact, we shall see that for our KH instability-induced flows involving weak fields (where reconnection topologies are formed on many scales) total energy dissipation is slightly "enhanced" when the grid is finer. This is opposite to what we expect from the effects of reduced numerical diffusion alone, but consistent with an increase in the number of reconnection sites, or " $X$ " points, allowed when smaller scale field structures can be resolved cleanly. Further, we find that for a fixed numerical resolution, addition of a very weak magnetic field substantially enhances the rate of energy dissipation, again as one expects in response to reconnection (e.g., Zimmer, Lesch, \& Birk 1997). Thus, we see strong evidence for unsteady, local reconnection as in high Reynolds number, MHD turbulence, but also that our numerical solutions are not quite converged in terms of total dissipation.

Below we will express all of our results in time units defined by the growth time of the linear instability, $t_{g}=$ $\Gamma^{-1}$, as estimated from graphs presented by Miura \& Pritchett (1982). That is, we express time as $\tau=t / t_{g}$. We find that especially the initial saturation of the instability, but also the relaxation processes are fairly uniformly expressed in these units. The time units, $t_{g}$, are listed for each case in Table 1, along with the duration of the simulation in units of $\tau$. The flows examined in the present paper all have $t_{g} \approx$ 1.6-1.7. For comparison the sound crossing time in the box is $t_{s}=L=2.51$ (since $c_{s}=1$ ) and the Alfvén wave crossing time is $t_{\mathrm{A}}=M_{\mathrm{A}} t_{s}$ (since $U_{0}=1$ ). The normalized turnover time for a large eddy is roughly $t_{E} \sim L /\left(U_{0} / 2\right) \sim 2 t_{s}$.

\subsection{Magnetic Field Evolution}

Since it enters prominently into our later discussions, it is helpful here to remind readers in a simple way of what we can expect for the local evolution of the magnetic field in these simulations. A full analysis of all the subtleties is beyond our scope here, so readers are referred to detailed discussions such as those by Moffatt (1978), Priest (1984), and Biskamp (1993). Equation (5) follows fields when the resistivity is exactly zero. As already mentioned, our finite difference code will introduce effects that mimic a finite resistivity, although it is not possible to define an exact value for the resistivity, $\eta$. The effective resistivity will also depend on grid resolution, decreasing roughly as $N^{-2}$ within smooth flows. Despite these limitations we can make good heuristic use of the resistive MHD extension of the induction equation (5). For our purposes it is interesting to cast that equation in the following form:

$$
\begin{aligned}
\frac{d \ln (|B| / \rho)}{d t} & =\frac{1}{2} \frac{d \ln p_{b}}{d t}-\frac{d \ln \rho}{d t} \\
& =\frac{B \cdot[(\boldsymbol{B} \cdot \nabla) \boldsymbol{u}]-\eta j^{2}+\eta \nabla \cdot(\boldsymbol{j} \times \boldsymbol{B})}{B^{2}},
\end{aligned}
$$

where $d / d t$ is the Lagrangian time derivative, the first term on the right represents field amplification by stretching, and the last two terms containing the resistivity account for magnetic "annihilation" and "diffusion." We have also used equation (2). The current density, $\boldsymbol{j}=\boldsymbol{\nabla} \times \boldsymbol{B}$. The term $\eta j^{2}$ is a dissipative term that balances the Joule heating in the analogous energy equation for the gas (e.g., Paper I). The last term is written as an expression involving the Lorentz force, $j \times B$, to show that it represents the transport of momentum flux in response to resistivity; i.e., the slippage of field lines.

A frozen-in field results when $\eta=0$. That leaves only the time derivatives and one term on the right of equation (6). If there is flow compression or expansion only perpendicular to $\boldsymbol{B}$ then the right side of equation (6) vanishes and leads to $|B| / \rho=$ constant or $p_{b} \propto \rho^{2}$. Those are the most common statements of field compression. However, more generally one needs to include the other ideal MHD term on the right that accounts for "stretching." In fact, field enhancements due to compression are much more limited than those due to stretching, especially in mildly compressive flows, such as those we are studying.

We also can see from equation (6) that resistive influences on magnetic energy are associated with both Joule heating and momentum transport. In fact reconnection leads to both irreversible heating of the local plasma and to its acceleration. The physics of reconnection is complex and beyond the scope of this paper. However, it may be helpful to estimate the dissipation rate using equation (6) and the SweetParker description of reconnection (e.g., Biskamp (1994); Parker (1994); Zimmer, Lesch, \& Birk 1997). Assuming an incompressible flow steadily carrying oppositely directed fields into a current sheet of thickness, $\delta$, equation (6) gives us a magnetic energy annihilation rate and associated dissipation rate per unit volume, $Q=\eta j^{2} \sim \frac{1}{2}(u / \delta) B^{2}$, where $u$ is the inflow speed. In this picture plasma flows out from the reconnection region at the Alfvén speed, so mass conservation leads to the relation between the current sheet thickness, $\delta$, and its width, $l=\delta\left(c_{\mathrm{A}} / u\right)$. Then, $Q \sim \frac{1}{2} B^{2}\left(c_{\mathrm{A}} / l\right)$. So, the integrated dissipation rate through reconnection depends on the field energy advected into reconnection sites and the summed volumes of all the reconnection sites. The current density within the current sheet can be estimated as $j \approx B / \delta$, so that the aspect ratio of the reconnection region 
is $\delta / l \sim 1 / N_{\mathrm{L}}^{1 / 2}$, where $N_{\mathrm{L}}=\left(l c_{\mathrm{A}}\right) / \eta$ is known as the Lundquist number of the plasma and is obviously related to the magnetic Reynolds number. Consequently, the dissipative volume scales as $l^{2} / N_{\mathrm{L}}^{1 / 2}$. In two dimensions, dissipative reconnection regions form out of tearing mode instabilities within a current sheet when the aspect ratio, $\delta / l$, is small (Biskamp 1994), and hence, when the magnetic Reynolds number is large. Thus, reconnection is not really steady, and the number of sites and their individual volumes will depend on the magnetic Reynolds numbers in the flow. Those are, indeed, the behaviors we see in our simulations.

\subsection{Issues}

We already alluded in the introduction to the basic character of nonlinear MHD KH instability properties found from previous work. Our intent here is to explore more fully the behaviors of weak magnetic fields in this situation. In preparation for that we note from past work several key features for two-dimensional symmetry:

1. When there is no magnetic field or if the field is orthogonal to the flow direction a shear layer will " role up." For the periodic flows considered here the result is a stable "cat's eye" vortex whose length equals the imposed periodicity on the space and whose height $\sim \frac{1}{3}$ the length. As long as the flow is subsonic or "submagnetosonic" no shocks are involved and the vortex decays only through viscous diffusion. We will not deal with the supersonic or supermagnetosonic cases here (for some work on those see, e.g., Pedelty \& Woodward 1991; Miura 1990, and references therein).In many astrophysical applications the kinetic Reynolds number of the flow is very large, so we wish to consider similar cases, so that dissipative decay times are long. For our simulations, the empirical viscous decay time of the flow is at least 4 orders of magnitude longer than the duration of our computations (see case 5 energy evolution curves in Fig. 3 below). Thus, the cat's eye represents a "quasisteady relaxed state," in which the shear layer has spread vertically by horizontal localization of vorticity and become stable. Of course, for nonperiodic systems there will be continued spreading due to additional vortex mergers, while in three dimensions the flows will be unstable to perturbations directed along the third direction. Those influences are beyond the scope of our present investigation, however.

2 . In the other extreme, if the magnetic tension force produced by a perturbation in the shear layer exceeds the "lift" force produced by the perturbation, the perturbed flow is stabilized. For wavevectors aligned with the flow that condition exists in a linear perturbation whenever the field is strong enough that $M_{A \|}=U_{0} /\left(B_{0} \cos \theta\right)<M_{A c}=2$. Then only viscous diffusion contributes to spreading of the original shear layer. As before, we can neglect that influence over finite times and describe the shear layer as remaining in a quasi-steady relaxed state from the start. For the present computations the critical magnetic field to stabilize a linear perturbation is $B_{c}=0.5$.For fields slightly weaker than critical, a small, but finite amplitude perturbation may lead to the same stabilization condition, perhaps after a small amount of quasi-linear growth to the instability. Such was the result obtained for the so-called "strong-field" case of Paper I, where $M_{\mathrm{A}}=2.5$. That calculation is listed as case 1 in Table 1, here. Under these conditions the flow is never far from laminar and there is a modest amount of spreading in the shear layer before it also reaches a quasi- steady relaxed state consisting of a broadened, laminar shear layer (a point made both in Paper I and by MBR). In either case 1 or case 2 , the total magnetic energy changed very little during the flow evolution. In Paper I we pointed out that for the symmetry imposed the mean vector magnetic field is time invariant; i.e., $\langle\boldsymbol{B}\rangle=$ constant, so any relaxed state with a relatively uniform field will automatically contain magnetic energy close to that of the initial conditions. This condition just reflects the conservation of magnetic flux on the grid. The "relaxed " magnetic energy is very slightly enhanced, because the field is not quite uniform at the end. A small amount of kinetic energy dissipation takes place in case 1 before the flow becomes relaxed. That amount is mandated by total energy conservation, the approximate magnetic energy conservation in this case, and the fact that on a fixed space the kinetic energy in a broad, symmetric shear layer is less than in a thin one. So, any evolution leading to a broadened, laminar shear layer requires an increased thermal energy determined by the final width of the layer, independent of how it got there. In our idealization the mass in the box is also exactly conserved, so the mean density is constant. Consequently, to first order there is no change in thermal energy by way of reversible, adiabatic processes. Most of the increased thermal energy must result from entropy-producing dissipation of some kind. Putting it simply, for these flows to relax entropy must be generated. These energy considerations apply to all of the calculations in our study if the relaxed state is a laminar flow. (It turns out that the kinetic energy of the cat's eye vortex is less than that for the initial flow, as well. So, its formation must also generate entropy.)

3. For initial fields too weak to prevent formation of the cat's eye vortex through magnetic tension, the shear layer will role up as for unmagnetized fluid flow. In Paper I, however, we saw that when the initial field is only a few times weaker than the critical value for linear stabilization, there follows a dramatic transformation in the flow as the cat's eye develops. Paper I considered a flow with $M_{\mathrm{A}}=5$. We list it in the current Table 1 as case 2 . As the vortex roles up in such cases, magnetic field lines are stretched around it, thus increasing magnetic energy at the expense of kinetic energy of the flow. The greatest magnetic pressures are produced in thin flux tubes formed between the vortex and its twins in periodic extensions of the space (see Figs. $4 a$ and 6 below). Especially within the vortex and around its perimeter, this evolution leads to magnetic reversals, beginning after about one turnover time for the vortex. The reversed fields are unstable to tearing mode reconnection. So, once that happens the magnetic field quickly reorganizes itself, releasing the magnetic stresses and stored magnetic energy. For case 2 this leads, as well, to disruption of the cat's eye and eventually to an almost steady, laminar flow, after formation and disruption of several weaker vortices. That, final, quasi-steady relaxed state, was similar to the initial conditions, except that the shear layer was broad enough to be stable against perturbations on scales that fit within the periodic box. The relaxed shear layer had a linear velocity profile (see MBR for similar points). There are additional interesting characteristics of the relaxed state. Remaining fluctuations in the magnetic and velocity fields were almost exactly correlated, so that they could be described as linearly polarized Alfvén waves. The magnetic energy returned to a level slightly above the initial conditions, with the final excess representing a pair of apparent "magnetic flux 
tubes" bounding a hot central core of the shear layer containing most of the entropy generated during the relaxation process. This final condition was reached by about $\tau=20$. The relaxed shear layer was broader in this case than case 1 (see also MBR), so that the kinetic energy was also smaller. Thus, even accounting for the slight increase in magnetic energy, we could correctly predict that case 2 with a weaker field was necessarily more dissipative than case 1 . That extra dissipation could come about only through the effects of reconnection. Other aspects of this case will be visited presently, since we will encounter them again in some new cases examined here.

Thus, from the calculations reported in Paper I and in MBR it is obvious that weak magnetic fields can play a major role in the evolution of MHD KH unstable flows. It is also apparent that these roles involve the exchange of energy and momentum from the gas to the magnetic field and then back to the gas through Maxwell stresses and reconnection. But it is not yet clear what are the crucial steps in that exchange, nor how it depends on the initial strength of the field, so that the ordinary $\mathrm{KH}$ behavior results, if it does, in the limit that the magnetic field becomes vanishingly small. In addition, although the linear MHD $\mathrm{KH}$ instability is not affected by the presence of a (possibly strong) field transverse to the flow (but still aligned to the plane of the shear), a field oblique to the flow can carry "circularly" polarized Alfvén waves. Since we found that the two-dimensional version of the problem generated linearly polarized Alfvén waves, it may be important to see if the nonlinear problem depends at all on the orientation of the field, or just on the strength of the field projected onto the plane as in the linear problem.

\section{RESULTS}

To meet the objectives at the end of the previous section we have carried out a set of seven new simulations. They are outlined in Table 1 as cases 3-9. (Once again, cases 1 and 2 were discussed in Paper I.) The new simulations were designed to cover a wide range of strengths for the magnetic field in the computational plane. They include flows that have exactly the same total field strength as case 2, but in which the initial field is oblique to the computational plane; i.e., $\theta \neq 0$ (cases 3,5 , and 6 ), as well as flows in which the field is entirely in the computational plane but is weaker than that in case 2 (namely cases $4,7,8$, and 9 ). The planeprojected field strength for cases 6 and 7 is an order of magnitude weaker than for case 2. Note that cases 3 and 4 are paired to have the same initial planar field strengths, as are cases 6 and 7 . This enables us to compare efficiently any distinct roles of field strength and orientation with respect to the plane. In case 5 the field is orthogonal to the plane, so we expect (and see) no important role for the magnetic field. As others have noted before, compressible influences are controlled in that case by magnetosonic waves rather than pure sound waves, so there is a very slight modification in response to that $\left[M_{m s}^{-1}=\left(M^{-2}+M_{\mathrm{A}}^{-2}\right)^{1 / 2}=1.02\right.$ in case 5]. We will not concern ourselves at all with flows in which the field is strong, by which we mean situations where magnetic tension precludes the nonlinear development of the MHD KH instability.

Figures 2 and 3 provide a broad overview of the evolution of the new models we computed. They illustrate the time variation of energy components (thermal, kinetic, and magnetic), as well as the pressure minimum ratio, $\beta_{\min }=$ $\left(p / p_{b}\right)_{\min }$, on the grid at each time. Figure 2 displays results for the runs that were computed with $N_{x}=512$, while Figure 3 shows the cases computed with $N_{x}=256$. In addition, to provide a sense of the influence of numerical resolution for very weak field cases both case $6 \mathrm{~h}\left(N_{x}=512\right)$ and case $6 \mathrm{~m}\left(N_{x}=256\right)$ are shown together in Figure 2. Resolution issues were discussed in detail for cases 1 and 2 in Paper I, where similar energy plots were also given. We shall add a few additional relevant comments below.

There is one distinctive detail about Figures 2 and 3 that is important to their interpretation. It was apparent to us by comparing animations of the important dynamical quantities that cases 3 and 4 were virtually indistinguishable from one another, and likewise for cases $6 \mathrm{~m}$ and 7 . Thus, the important issues are somewhat easier to see if we eliminate $B_{z}$ to first order in Figures 2 and 3. We plot a reduced energy for the cases with $\theta \neq 0$. The magnetic and kinetic energy plotted include only the planar components; i.e., $E_{b}^{\prime}=\int(1 / 2) B_{p}^{2} d x d y$, where $B_{p}=\left(B_{x}^{2}+B_{y}^{2}\right)^{1 / 2}$, and $E_{k}^{\prime}=$ $\int(1 / 2) \rho\left(u_{x}^{2}+u_{y}^{2}\right) d x d y$. To compensate, the total energy is also reduced as $E_{\mathrm{Tot}}^{\prime}=E_{b}^{\prime}+E_{t}+E_{k}^{\prime}$, where $E_{t}$ is the thermal energy. Thus, we ignore the almost constant energy contributions from $B_{z}$ and $v_{z}$. Note, however, it is the total energy, not the reduced total energy, that is conserved. In the definition of $\beta_{\min }$, total magnetic pressure from all three components was used, since it is the entire pressure that exerts a force on the plasma.

We can see that the reduced energy evolution in cases 3 and 4 are almost identical in Figure 2, and the same is true of cases $6 \mathrm{~m}$ and 7 in Figure 3. Thus, we observe in $2 \frac{1}{2}$ dimensions, at least, that the transverse magnetic field component $\left(B_{z}\right)$ plays no significant role in nonlinear evolution of the instability. The rationale for some role comes from the observation that finite $B_{z}$ and $v_{z}$ enable circularly polarized Alfvén waves in $2 \frac{1}{2}$ dimensions, while only linearly polarized Alfvén waves are allowed in two dimensions. In other words, there are twice as many degrees of freedom in $2 \frac{1}{2}$ dimensions for Alfvén waves to help disperse perturbations. However, there are two arguments to support our observation of a minimal role. First, the group velocity vector for Alfvén waves along which physical information propagates is $v_{g}=B / \rho^{1 / 2}$; i.e., aligned with the magnetic field (see, e.g., Landau, Lifshitz, \& Pitaevskii 1984). With invariance of quantities along the $z$-direction, only the group velocity components projected onto the $x-y$ plane are relevant, and they are independent of $B_{z}$. The second point is that even though $B_{z}$ and $v_{z}$ are finite in $2 \frac{1}{2}$ dimensions, the above symmetry restricts their contributions to those of magnetic pressure (see eqs. [2]-[5]); i.e., to the total pressure gradient and the fast and slow wave speeds. In the present case with weak fields, even that has little importance. For example, the evolution of $\beta_{\min }$ is almost the same for cases 3 and 4 , where stretching of the field in the plane is dominant in case 3 . For cases $6 \mathrm{~m}$ and 7 the values of $\beta_{\text {min }}$ are always large, because neither the planar nor the transverse fields are very strong. But at $\tau \sim 5$ when the stretching of the planar field is greatest, the values of $\beta_{\min }$ are still similar. So, our results indicate that for weak fields the extra degrees of freedom allowed in $2 \frac{1}{2}$ dimensions have no significant effect on the KH instability. These flows are essentially two-dimensional.

In one of the most striking findings from these simulations, we find two distinctive evolutionary behaviors for 

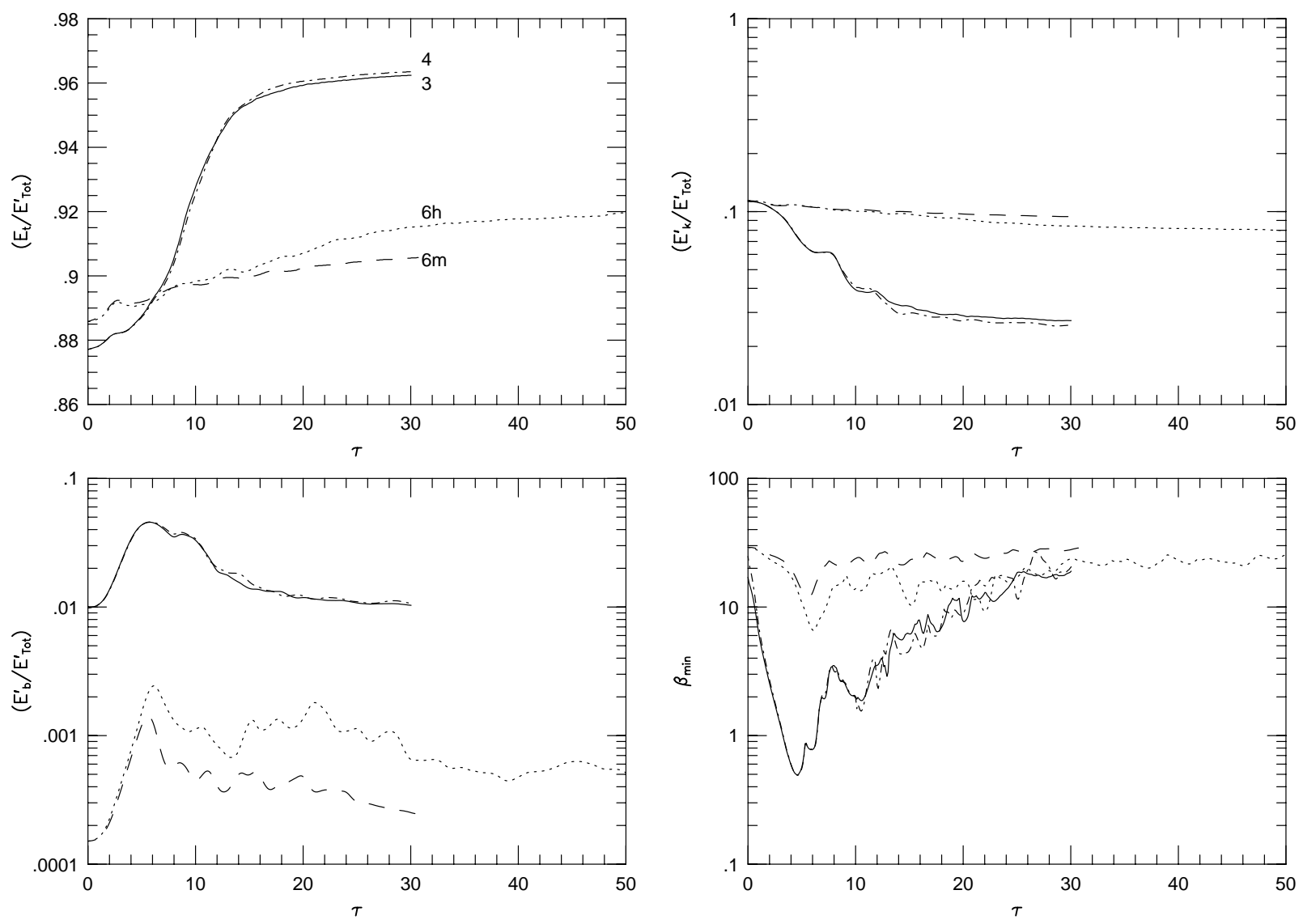

Fig. 2.-Time evolution of the high-resolution simulations (cases 3, 4, 6h), plus the medium resolution simulation case $6 \mathrm{~m}$. Shown are the normalized total thermal, kinetic, and magnetic energies, as well as the minimum value of the plasma $\beta$ parameter at each time. To emphasize the minimal importance of the transverse magnetic field component, $B_{z}$, "reduced" energies are shown that exclude $B_{z}$.

weak field flows, depending on the field strength. When the planar magnetic fields are very weak, it turns out that the evolution of the two-dimensional MHD KH instability is qualitatively similar to the gasdynamic version of the instability. That is, the cat's eye vortex continues to exist as long as we extend the simulation. The magnetic field does, through unsteady reconnection, substantially enhance energy dissipation of the vortex over that from the gasdynamical case, however. This can be seen by comparing case $5\left(B_{p}=0\right)$ in Figure 3 with case $6 \mathrm{~m}$ or case $7\left(B_{p}=\right.$ 0.02 ). We will characterize such flows as having "very weak fields," and the role of the field as "dissipative." On the other hand, if the initial fields approach the critical field for linear stabilization within a factor of a few, the cat's eye is disrupted and the field causes the flow to reorganize into a laminar form (as in case 2). We call this simply the "weakfield " regime, and the field "disruptive." In either situation the magnetic energy peaks about the time of the initial reconnection instability. For the disruptive cases there is a fairly prompt return of the magnetic energy near to its initial value (see Figs. 2 and 3). In effect, the magnetic field plays the role of a catalyst, storing energy temporarily and through it modifying the plasma flow. That role is fairly dramatic, since reconnection leads to dynamical alignment and self-organization in the flow. For dissipative cases, the magnetic energy declines much more slowly, and in the high-resolution case $6 \mathrm{~h}$, seems mostly to oscillate. That behavior results from the fact that the cat's eye vortex continues to capture magnetic flux, amplifying it and thence dissipating it.
The existence of two qualitatively distinct evolutionary patterns is also quite apparent in the histories of the thermal and kinetic energies displayed in Figures 2 and 3. The kinetic energy decay in case 6 is almost exponential, with a time constant that can be roughly estimated as $\tau_{d} \sim 10^{4}$. By contrast, cases 3 and 4 show before $\tau \sim 10$ a sharp drop in $E_{k}^{\prime}$ along with an accompanying increase in $E_{t}^{\prime}$, followed by a slow, possibly exponential evolution similar to case 6 . For all cases in Table 1 the magnetic energy is always small (although it can briefly increase by factors between 6 and 20 it never contributes more than a few percents to the total energy), so on the face of it the magnetic energy would not seem to be important. In fact, it can be crucial, as we have already outlined and will discuss more fully, below. Figure 3 , which shows a wider range of projected field strengths, shows similar patterns with some variation in the abruptness of the early energy transition. We also conclude that as $B_{p} \rightarrow 0$ the evolution of the two-dimensional MHD KH instability will smoothly approach that for ordinary gasdynamics.

\subsection{Very Weak Fields: Dissipative}

Let us now describe in more detail the characteristics of the two weak-field regimes and also establish the physical boundary between them. Case 6 and case 7 had the weakest finite planar field, $B_{p o}$, that we considered, and they demonstrate very weak field patterns. Figure 4 summarizes flow properties for case $6 \mathrm{~h}$ at times $\tau=5$ and 30. Figure $4 a$ shows the flow vorticity component out of the plane, $\omega_{z}=$ $(\nabla \times u)_{z}$, while Figure $4 b$ shows the magnetic field lines as 

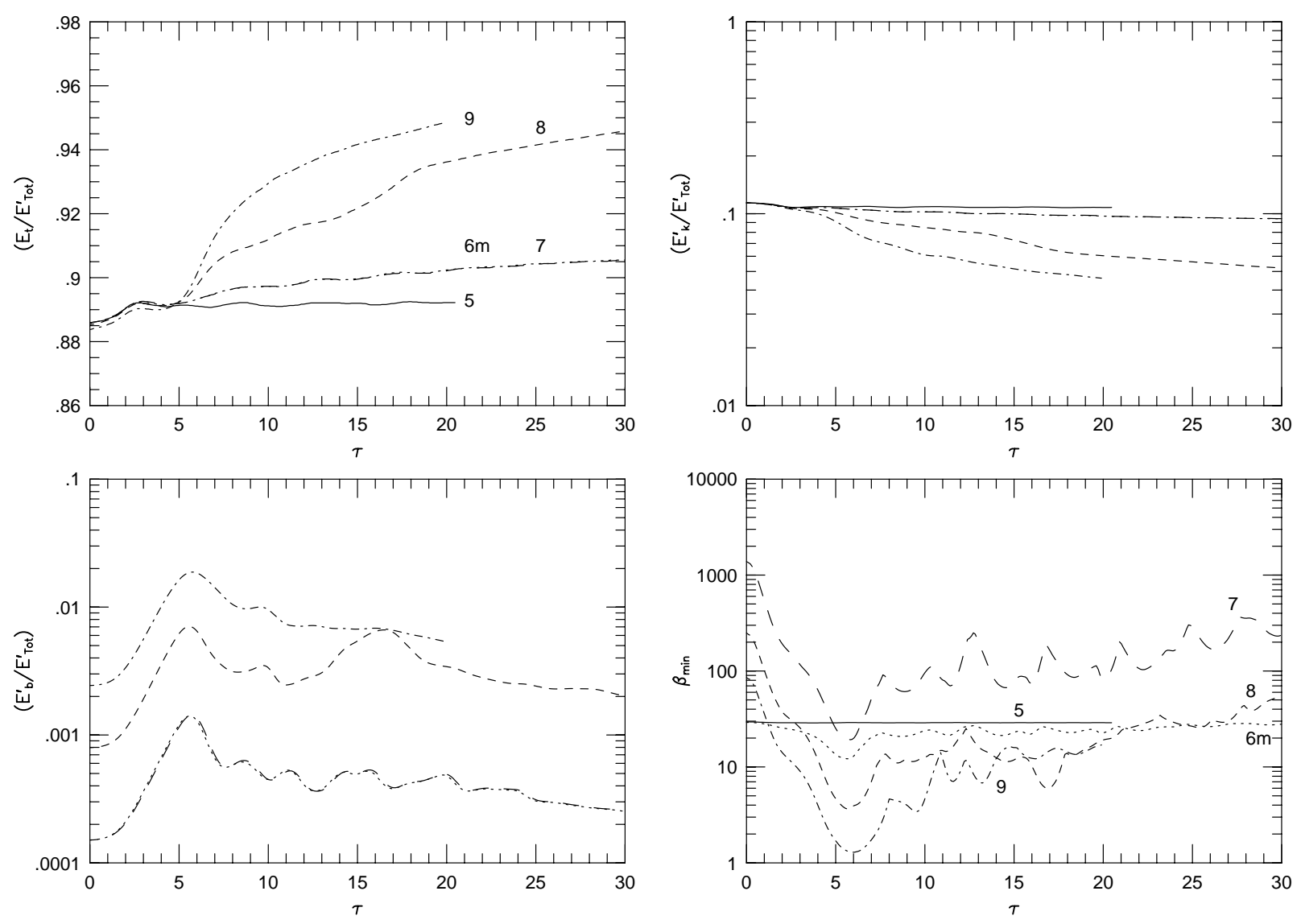

FIG. 3.-Same as Fig. 2, except for medium resolution simulations (cases 5, 6m 7, 8, 9)

they project onto the plane. Except for minor fine structure that aligns with the planar field, the vorticity at $\tau=5$ is the same as for a flow with $B_{p}=0$ of case 5 . It also is fairly close to that for all our simulations, except case 1 at this early time. Primarily, this image illustrates the formation of the cat's eye and how that concentrates vorticity. Note that the "corners" of adjacent cat's eyes overlap, with a shear layer between them. It is in that shear layer where magnetic fields are most strongly enhanced, as seen for this time in Figure $4 b$. Extensions of that feature around the perimeter also contain concentrations of magnetic flux. These regions represent flows where gas is accelerated out of a stagnation point midway between vortex centers. Frozen-in fields are thus pulled out or "stretched" and amplified. At $\tau=30$, the cat's eye vortex is still largely the same, except for some complex, low-level vorticity structures outside the main vortex. Their origins are made clearer by examination of the magnetic field structure at this time. Those same regions outside the cat's eye also contain isolated magnetic flux islands and field reversal regions. Such magnetic features reveal an environment where magnetic reconnection is active. The relationship is that reconnective processes not only reorganize the magnetic field topology and release magnetic energy, but they also accelerate the local plasma, and that contributes to the local vorticity. By contrast to case 2 (or as we shall see cases 3 and 4 , as well), however, magnetic stresses in cases 6 or 7 produce only small modifications to peripheral flows and are far too weak to disrupt the cat's eye.

The vortex interior shows initial signs in Figure $4 b$ of magnetic flux expulsion by $\tau=5$, a well-known phenome- non (e.g., Weiss 1966). At this early stage, just as the vortex is fully formed, there is still some magnetic flux that threads through the "eye." That does not seem to be the case at the later time. In a close examination of field structures within the vortex we can find no evidence after about $\tau=10$ that any magnetic flux threads the vortex. Instead, the field breaks into flux islands within the vortex, and those are annihilated through mergers. This is, of course, just what high Reynolds number (both kinetic and magnetic), nonlinear resistive MHD flows are expected to do in response to reconnection (e.g., Biskamp 1993, 1994). The field structure in the bottom panel of Figure $4 b$ is qualitatively very similar to that found by Weiss (1966) from a classic passive field simulation in a steady vortex with magnetic Reynolds number $R_{m}=10^{3}$, but very different from that for small magnetic Reynolds number $\left(R_{m}=20\right)$. Our own estimates of effective magnetic Reynolds numbers in the case $6 \mathrm{~h}$ simulation give numbers in excess of $10^{3}$ (Ryu et al. 1995; Frank et al. 1996), so the comparison is very reasonable.

We emphasize, however, that the field behaviors seen here, and presumably by Weiss, result from localized, inherently time-dependent reconnection, not simple flux diffusion. To demonstrate that we compute in the Appendix the equilibrium passive magnetic fields for a simple vortex in a resistive fluid. Figure 5 displays two examples of magnetic field structures predicted by this steady state resistive MHD theory. The velocity structure for this model vortex is similar to that observed in the simulated flows for case 6 . The core, $r<r_{0} / 2$, has a constant vorticity, $A$ (see Fig. $4 a$ ), while there is an outer flange in which the velocity decreases to zero at $r=r_{0}$. Solutions depend only on the effective 

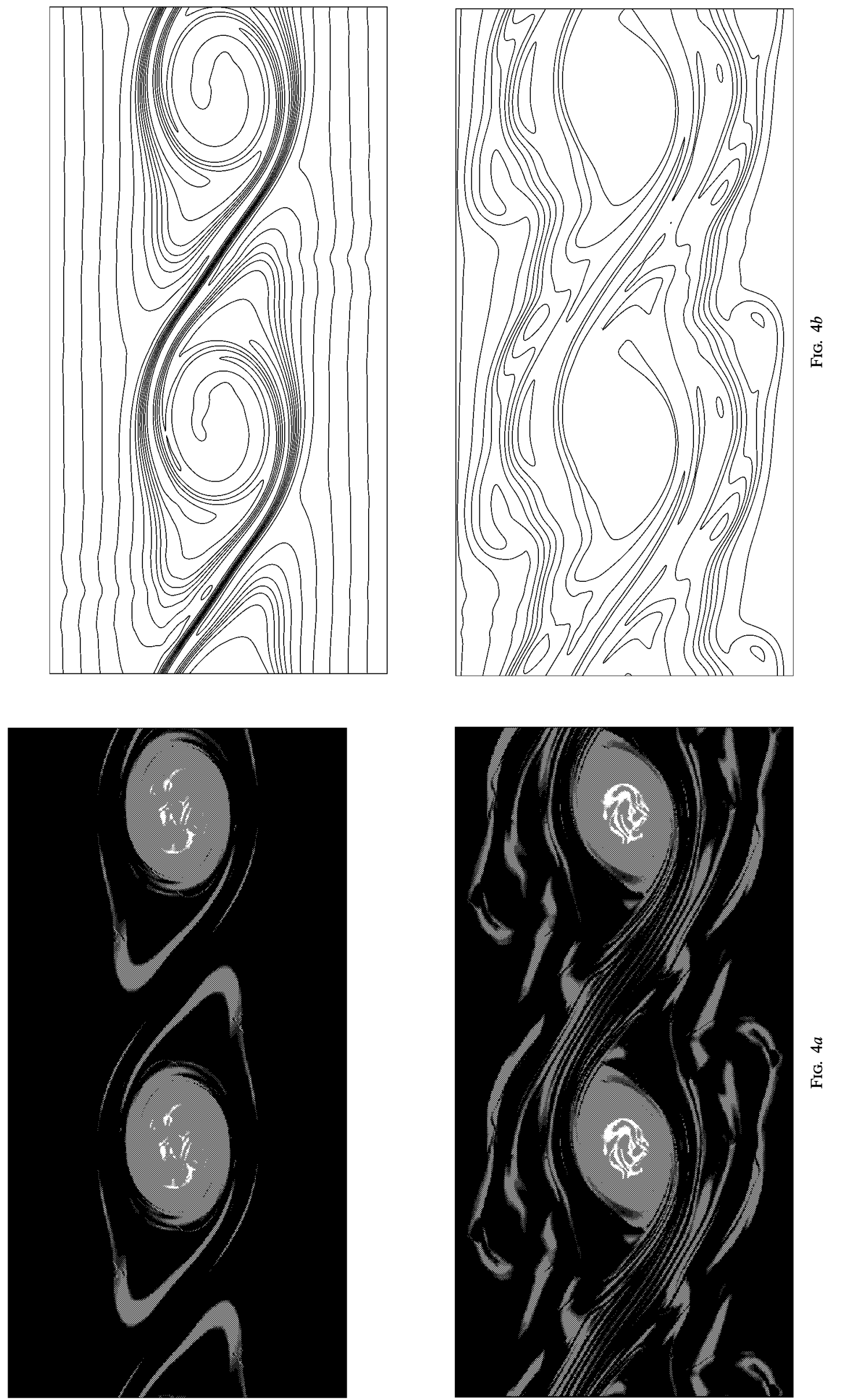

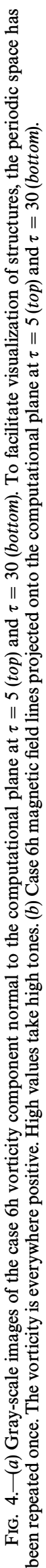




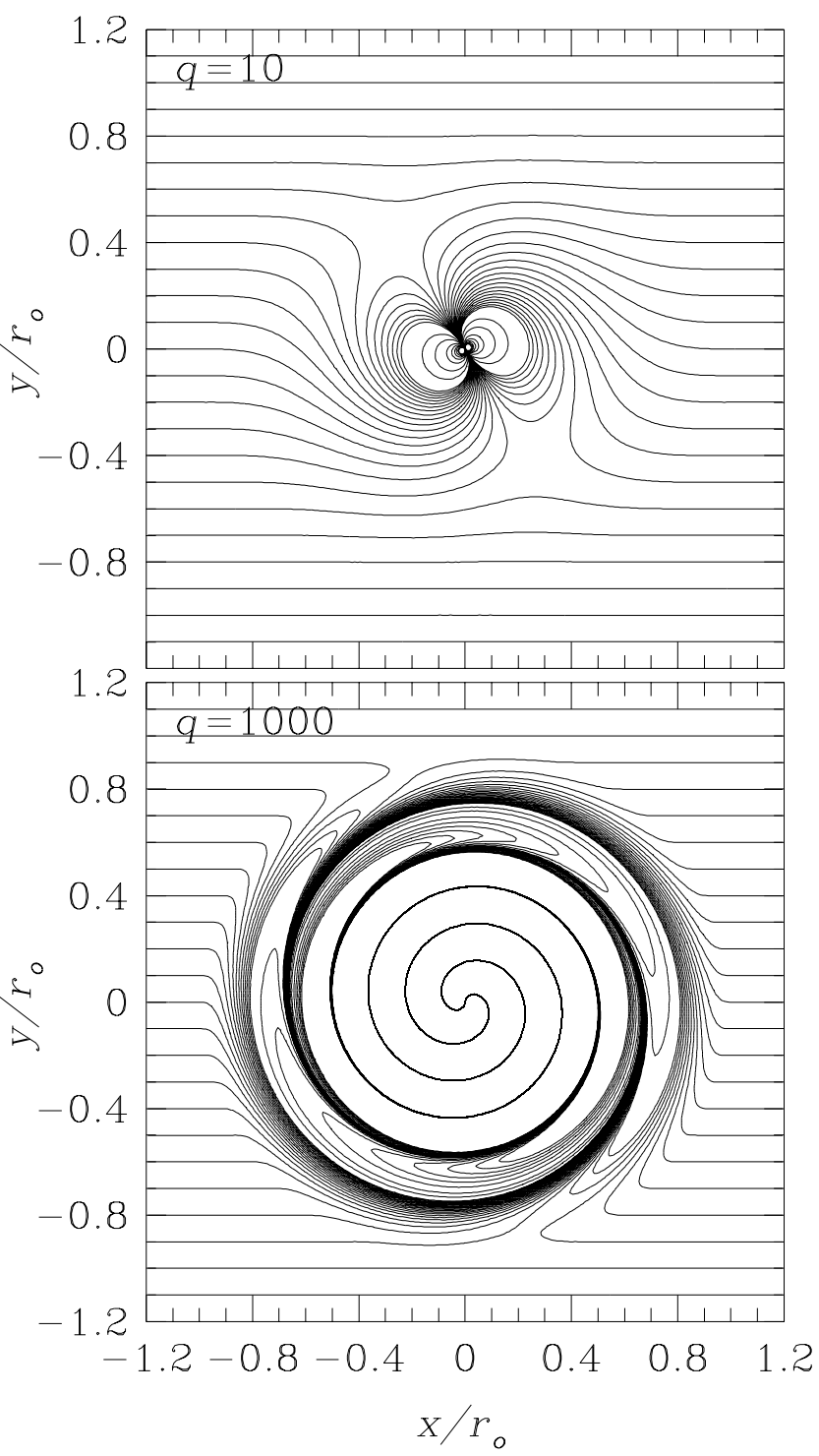

FIG. 5.-Magnetic field lines from an analytical model to study diffusive flux expulsion from a steady eddy with $q=10$ (top) and $q=1000$ (bottom). See the text for the definition of $q$.

magnetic Reynolds number, $q=2 A r_{0}^{2} / \eta$, within the vortex. This parameter roughly measures the ratio of the timescale for magnetic field diffusion to the rotation period of the vortex. When $q$ is a few $(q=10$ in the top panel of the figure), flux diffuses quickly enough to almost flatten out field lines to their external pattern except in the vortex core. At the center field lines are actually concentrated by rotation into a quasi-dipole pattern. That dipole results because reconnection into a simpler topology is not permitted.

The steady state pattern is very different when $q$ is large, since field is almost frozen into the vortex. Above $q \sim 10^{2}$ the field forms a spiral pattern in the vortex core whose pitch depends on $q$. Shear is greatest in the flange region of this vortex and flux becomes concentrated increasingly into the vortex perimeter. That particular behavior does resemble case 6 in some ways. There are multiple field reversals in the perimeter, with that number determined in the steady state calculation by the balance between field diffusion and addition of new turns to the field through rotation. The bottom panel in Figure 5 shows the steady state solution for $q=10^{3}$. Despite some superficial similarities, there is an obvious and essential difference between the truly steady state solution in Figure 5 and the "quasi-steady" field in the two time-dependent simulations (ours and Weiss's). For the steady solutions the field line that was originally through the vortex center remains and is wrapped into a spiral pattern within the core giving an almost constant field strength there. In the time-dependent cases, reconnection changes the field structure inside the vortex. The same statement applies to Weiss's simulation. If the effective magnetic Reynolds number within the flow is large enough for topologies to develop that are tearing-mode unstable, field within the vortex "breaks" off from the external field and is destroyed. Thus, we find good agreement with Weiss in the nature of eddy flux expulsion and see that it cannot be viewed as a steady magnetic diffusion.

The value of $B_{p}$ never drops to zero inside the vortex of any of our very weak field cases, although it is as much as 2 orders of magnitude smaller than along the vortex perimeter. Action around the perimeter episodically injects new magnetic flux into the vortex interior, so this process continues as long as we have followed the flow. Examination of the magnetic energy, $E_{b}^{\prime}$, for case 6 in Figure 2 shows related, episodic peaks on rough intervals, $\Delta \tau \sim 2.5-3$. That corresponds to about half a turn over time for the vortex, and represents the interval on which fields along the vortex perimeter are stretched until they become "folded," so as to make them subject to tearing mode reconnection. So, whatever flux is caught in this flow becomes amplified, increasing the magnetic energy, before reconnection rearranges the field lines. Those lines outside the vortex are relaxed toward the initial field configuration, often through multiple reconnection events, while those inside are isolated into closed islands. Note that the total magnetic flux threading our box is exactly conserved and exactly zero in these computations. Thus, any field line entering on the left boundary must at all times extend continuously to the right boundary or exit again on the left. Closed flux islands can exist, but nothing prevents them from being destroyed, since they contribute no net flux.

Reconnection, and most obviously flux-island destruction, is irreversible, so that it must be accompanied by entropy generation. That outcome is very apparent in Figures 2 and 3. Recall that to first-order total thermal energy changes in these, closed-system simulations reflect nonadiabatic processes. Compare first the evolution of thermal energy in cases $6 \mathrm{~m}, 7$, and 5 . We see that after some viscous dissipation necessary to form the cat's eye, the case 5 with $B_{p 0}=0$ has almost constant total thermal energy to the end of the simulation. By contrast cases $6 \mathrm{~m}$ and 7 show a steady rise in $E_{t}$, with small amplitude oscillations associated with major reconnection events as described above. Thus, the main dynamical impact of the field is enhanced dissipation. The total magnetic energy remains small in all cases, but for the dissipative cases that reflects a near balance between the rate at which kinetic energy is being transferred to magnetic energy and the rate at which magnetic energy is being dissipated. For example, from initial field energy evolution in Figure 3 we can crudely estimate for case $6 \mathrm{~m}$ that magnetic energy is generated at a rate $d E_{b}^{\prime} / d t \sim 3 \times 10^{-4}$, which is very close to the mean slope of the thermal energy curve, $E_{t}$.

Looking next at the case $6 \mathrm{~m}, \mathrm{~h} E_{t}$ plots in Figure 2, we see that energy dissipation is greater in the higher resolution case $6 \mathrm{~h}$. That results despite a smaller numerical resistivity 
for the higher resolution simulation. This is because in case $6 \mathrm{~h}$ a larger amount of flux is caught in the vortex, so more reconnection sites develop. We estimate that $\sim 10 \%$ of the total magnetic flux is attached to the vortex at the end of the calculation $(\tau=50)$ in case $6 \mathrm{~h}$, while only $\sim 3 \%$ is attached at the end of the calculation $(\tau=30)$ in case $6 \mathrm{~m}$. As the effective magnetic Reynolds number increases there is a tendency for decreased resistivity to be countered by an increase in the number of reconnection sites, as our earlier discussion of reconnection theory would suggest. If we compare simple estimates for rates of magnetic energy generation for cases $6 \mathrm{~m}$ and $6 \mathrm{~h}$ to the rates of thermal energy increase, we see that they are consistent, as we found earlier for case $6 \mathrm{~m}$, alone. The increased dissipation for the higher resolution simulation is relatively modest, however. Some studies of resistive MHD turbulence suggest, in fact, that at very large Reynolds numbers total reconnection rate in a complex flow will be insensitive to the value of the resistivity (Biskamp 1993, 1994). If confirmed more generally, that could provide a practical measure of convergence in studies of the present kind.

\subsection{Weak Fields: Disruptive}

Looking again at thermal energy evolution in Figures 2 and 3, we can see a clear behavioral transformation in the sequence: case $5 \rightarrow 6(7) \rightarrow 8 \rightarrow 9 \rightarrow 3(4)$. This grouping is arranged in order of increased $B_{p 0}$. There is a sharp rise through the sequence in the amount of dissipation associated with the initial formation of the cat's eye vortex, as well as a more modest increased slope to the subsequent longterm dissipation rate. The physical character of this transformation is apparent if one compares Figure $4 b$ with Figure 6. The latter shows for case 3 the magnetic pressure distribution at three times, $\tau=5,10$, and 30. Behaviors for this simulation are qualitatively similar to case 2 , as discussed in detail in Paper I and outlined in $\S 2.3$. Here we note that, while the field appears wrapped around the vortex at $\tau=5$, it has a laminar appearance at $\tau=30$. The velocity field undergoes a similar transition; i.e., the vortex is completely disrupted. The magnetic field at the intermediate time, $\tau=10$, shows aspects of both the other times. Curiously, however, the flow in the dominant vortex pattern there has the opposite vorticity to the original flow. That feature is short-lived. We can understand this flow transition from rotational to laminar by examining Figures 7 , 8, and 9, also relating to case 3 . In Figure 7 we show the $\log$ of $\beta=p / p_{b}$ with magnetic field lines overlaid at $\tau=5$. The minimum $\beta=0.55$ in the strong flux tube connecting vortices, but the magnetic field is dynamically significant most of the way around the perimeter of the vortex. The maximum $\beta \sim 10^{6}$ in small regions where magnetic reconnection has begun and the field strength has decreased to very small values.

To understand how the magnetic field disrupts the vortex consider the forces involved. The centripetal force associated with motion around the cat's eye is $\rho u_{\phi}^{2} / R \sim \frac{1}{2} \rho U_{0}^{2} \sim$ $\frac{1}{2}$, since we observe that $u_{\phi} \sim 1 / 2^{1 / 2}$ and $R \sim 1$ for the vortex. Indeed, we also confirm that when $B_{p 0}=0$, the pressure gradient force within the vortex $-\nabla p \approx 0.5$ and is directed toward the vortex center, so that it supplies the necessary force. On the other hand, in Figure 8 we display the magnetic field lines along with vectors representing the magnetic tension force; i.e.,
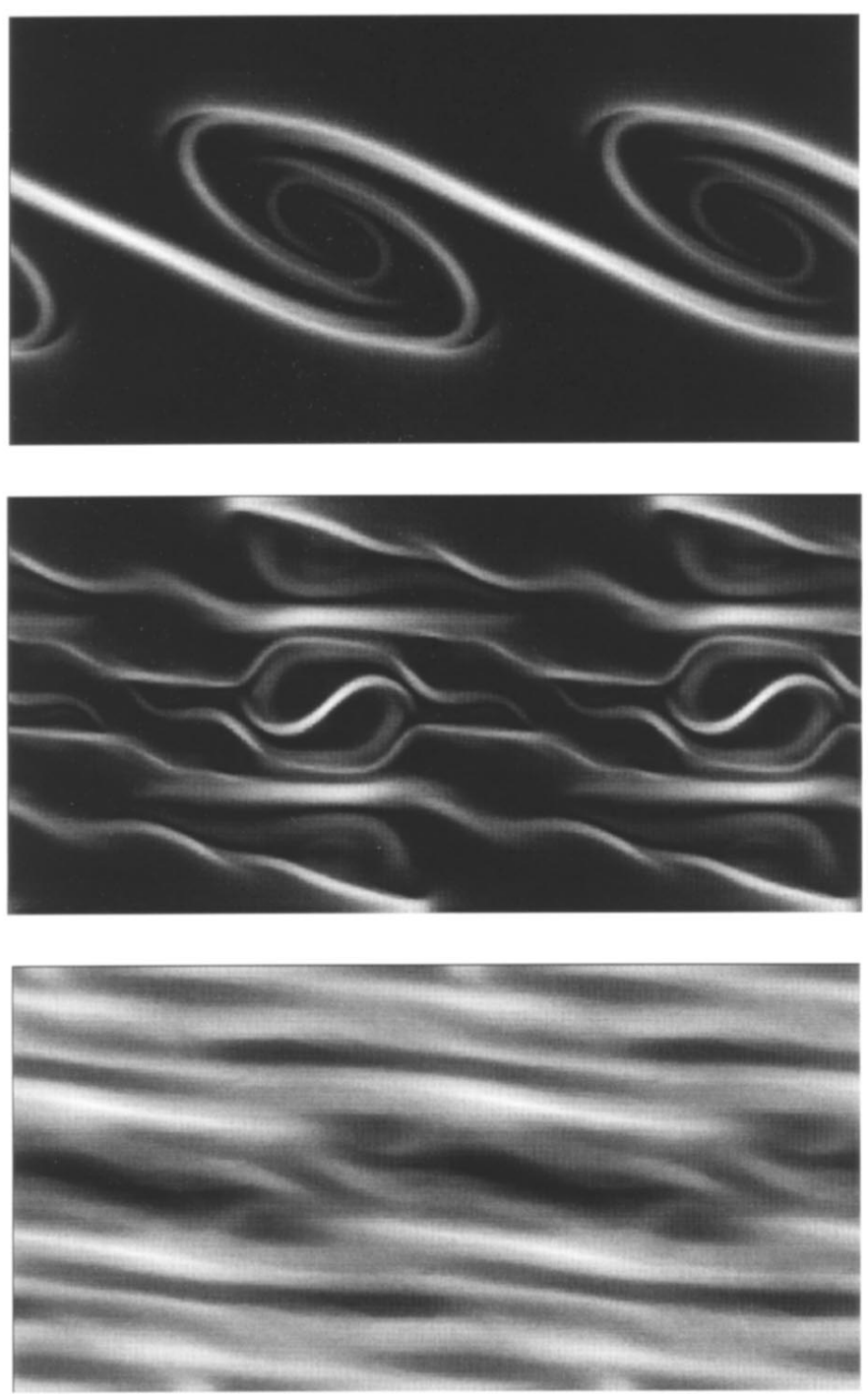

FIG. 6.-Gray-scale snapshots of the magnetic pressure distributions for case 3 at $\tau=5,10$, and 30 , showing the stages in disruption of the cat's eye vortex by the magnetic field. High values take high tones.

$$
\boldsymbol{T}=(\boldsymbol{B} \cdot \nabla) \boldsymbol{B},
$$

at $\tau=7.5$ for case 3 . It is apparent that magnetic tension forces are concentrated where the field has been pulled into loops by the flow and that they are directed toward the center of the vortex. The peak value of $|T|=1.85$. At $\tau=7.5$ in case 3 , however, the total pressure gradient is small and actually of the wrong sign to effect significantly the motion of the plasma. The total force vector field is very similar to the tension force field shown in Figure 8. At the same time "X-points" in the field topology show that reconnection is underway that will isolate the associated field lines. Subsequently, flux islands are formed along the "axis" of the cat's eye, and the magnetic tension pulls the plasma frozen into those loops toward the original center of the vortex, disrupting its rotation. Field line segments reconnecting outside the vortex core will tend to relax toward the original field topology.

These observations allow us to estimate simply what minimum initial field, $B_{p 0}$ should lead to vortex disruption. Since the early evolution of a weak field is self-similar, we 


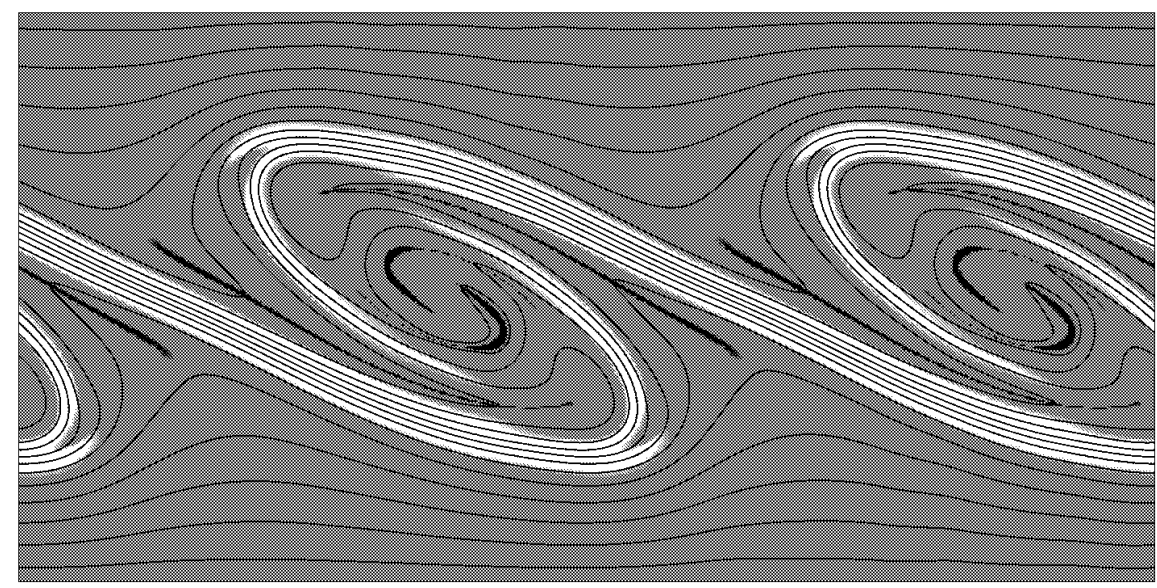

FIG. 7.- Inverted gray-scale image of $\log \beta=p / p_{b}$ for case 3 at $\tau=5$. Projected magnetic field lines are overlaid. The minimum $\beta \sim 0.55$ in the strong flux tube between vortices, while the maximum $\beta \sim 10^{6}$ near the center of the cat's eye.

can use the behavior from case 3 to estimate $|T| \approx$ $1.85\left(B_{p 0} / 0.14\right)^{2}$ at the time of the first major reconnection in all weak-field cases. Our earlier discussion requires $|T| \geq$ $\frac{1}{2} U_{0}^{2} \rho=\frac{1}{2}$ for disruption, leading to the constraint $B_{p 0} \geq$ 0.05 , or alternately, $B_{p 0} \geq 0.1 B_{c}$, where $B_{c}$ is the critical field for stabilization of original instability. Indeed, as Figure 3 demonstrates, the transformation between dissipative and disruptive evolution occurs for conditions between those of cases 8 and 9; i.e., for initial field values between 0.04 and 0.07 .

Figure 9 illustrates why the vortex disruption process is also highly dissipative. The top panel shows at $\tau=7.5$ in case 3 the gas entropy distribution, while the lower panel displays the electric current density, $|j|$ with the field lines overlaid. From this we can see that excess entropy is concentrated into regions where reconnection is currently active (highlighted by $|j|$ ) or recently active. Ohmic heating $\left(\propto j^{2}\right)$ is partly responsible for the irreversible energy exchange. The remainder should be viscous dissipation of small-scale, disordered motions. As we discussed in Paper I, the final laminar flow that results in this class of flow includes a central sheet of hot gas containing most of the excess entropy produced through the self-organization of the flow.

\section{SUMMARY AND CONCLUSION}

We have carried out a series of high-resolution MHD simulations of Kelvin-Helmholtz unstable flows in $2 \frac{1}{2}$ dimensions. All of these simulations involve magnetic fields initially too weak to stabilize the flows in the linear regime; i.e., $B_{p 0}<B_{c}$. Thus, since simulations are performed on a periodic space, flows all begin formation of a single "cat's eye" vortex. If the field lying in the computational plane is absent or "very weak" the cat's eye structure becomes a persistent, stable feature that represents a "quasi-steady" equilibrium. When there are "very weak" magnetic fields in the plane they become wrapped into the vortex and amplified by stretching. However, within a single turn of the vortex they are subject to tearing mode instabilities leading to magnetic reconnection. That reconnection isolates some magnetic flux within the vortex, which is eventually annihilated. This is the process through which flux is effectively expelled from a vortex. As long as the vortex persists this process will repeat. Since reconnection is irreversible, this process is also dissipative and leads to an increase over viscous effects in conversion from kinetic to thermal energy. We find in this regime that as the initial magnetic field within the computational plane is increased the dissipation

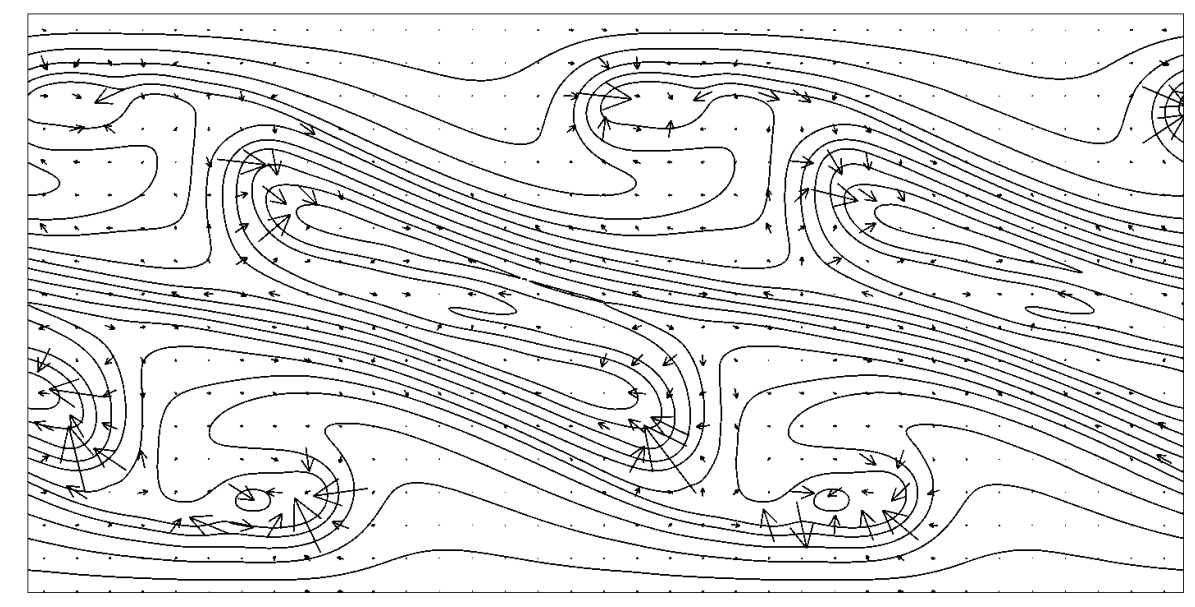

FIG. 8.-Magnetic field structure for case 3 at $\tau=7.5$, just as substantial magnetic reconnection is underway. Projected field lines are shown, along with arrows that represent magnetic tension forces. The maximum magnetic tension force is 1.74 . 

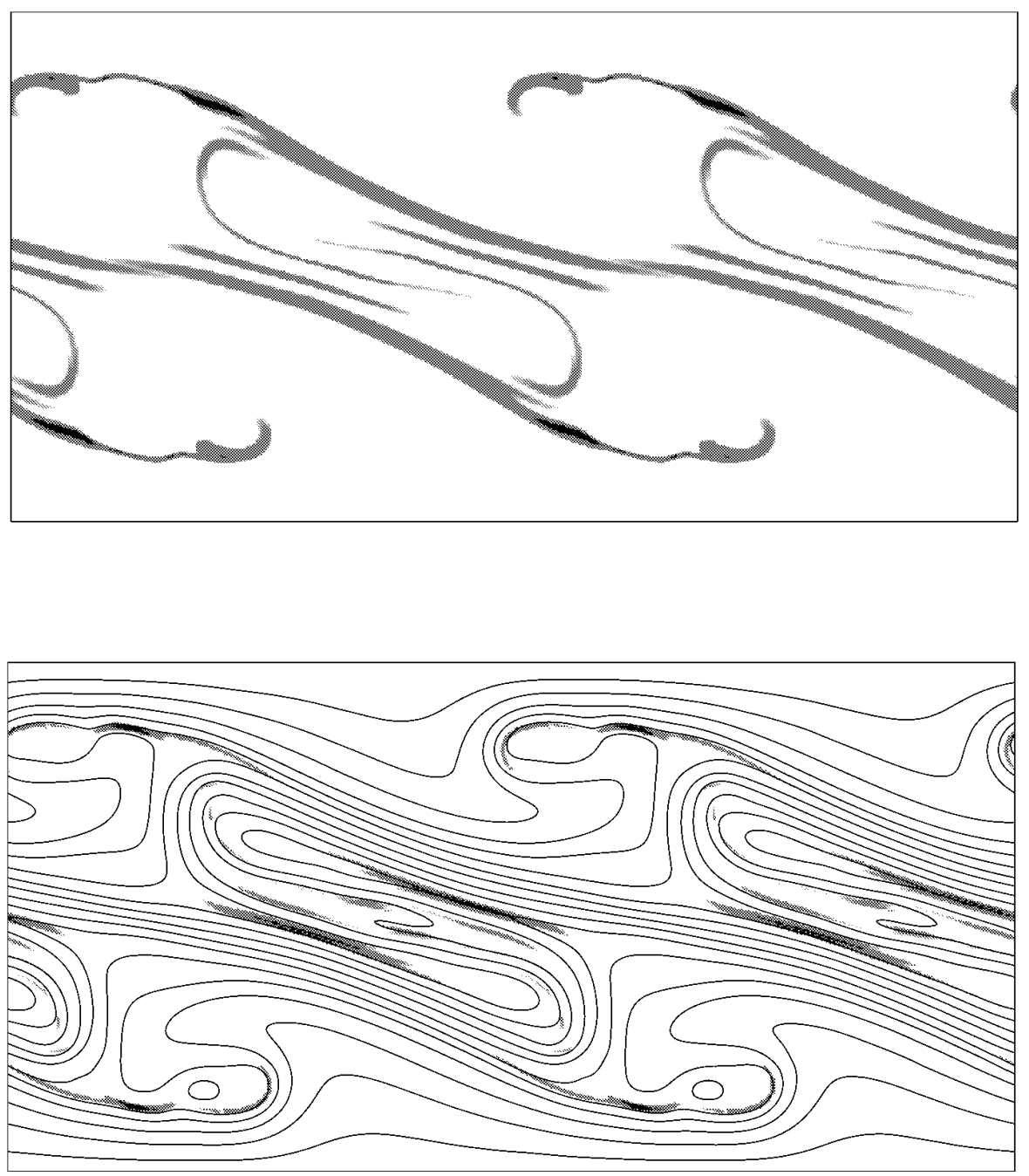

Fig. 9. - Inverted gray-scale image of the gas entropy (top) and electrical current (bottom) distributions for case 3 at $\tau=7.5$. Magnetic field lines are also laid on top of the current distribution to emphasize the relationships. High values take low tones.

rate increases in a similar manner. Likewise, as we use a finer numerical grid, thus reducing the effective numerical resistivity and viscosity, the dissipation rate increases, reflecting the increased ability of our code to capture small-scale reconnection events. This trend is backward from what one would expect if simple magnetic diffusion were primarily responsible for the reconnection. It suggests, perhaps that if we had been able to extend these calculations to even higher resolution the energy dissipation rate might have converged to a value independent of the effective resistivity, just as some studies of resistive MHD turbulence find. The reconnection and expulsion of flux within vortices in our simulations are similar to those in a classic study by Weiss of vortex flux expulsion in large-magnetic-Reynolds-number flows.

If the initial magnetic field is strong enough that within a single turn of the vortex it is amplified around the vortex perimeter to "dynamical" strength $\left(B^{2} \sim \rho U^{2}\right)$, then the reconnection described in the previous paragraph releases stresses that are capable of disrupting the vortex entirely. This can happen in a single event or, if the field is only marginally strong enough $\left[B_{p 0} \sim(1 / 10) B_{c}\right]$, through a succession of dynamical realignment events. In either case the net result is a laminar but marginally stable flow in which the original shear layer is greatly broadened. Thus, as we discussed fully in Paper I, such fields can have a remarkable stabilizing influence. This is despite the fact that their total energy content is a minor fraction of the total, so that they are nominally too weak to be important, according to the usual criteria.

We considered cases in which the magnetic field was entirely within the flow plane and others in which the field was oblique to that plane, in order to examine the role in nonlinear flows of the component out of the plane. For the $2 \frac{1}{2}$-dimensional flows we have studied, only the field components in the flow plane have any dynamical significance. In fully three-dimensional flows, however, we expect further evolution of the "quasi-steady relaxed states" of both very weak field (or dissipative) cases and weak field (disruptive) cases. The cat's eye vortex of very weak field cases is subject to a three-dimensional instability known as the elliptical instability (Pierrehumbert 1986; Bayly 1986) unless the flow lines around the vortex follow perfect circles. The planar shear flow of weak field cases is stable against linear perturbations but unstable to three-dimensional finite-amplitude perturbations (Bayly, Orszag, \& Herbert 1988). Thus, it will 
be important to extend the present study to the fully threedimensional regime, and we are preparing to do that.

This work by T. W. J., J. B. G., and A. F. was supported in part by the NSF through grants AST 93-18959 and INT-
9511654, through NASA grants NAGW-2548 and NAG 5-5055 and by the University of Minnesota Supercomputer Institute. The work by D. R. was supported in part by Seoam Scholarship Foundation. We are grateful to B. I. Jun for stimulating and helpful discussions about these results.

\section{APPENDIX A}

\section{DIFFUSIVE FLUX EXPULSION FROM A STEADY VORTEX}

In order to show that the flux expulsion from vortex shown in Figure $4 b$ requires localized, unsteady reconnection, not just diffusion, here we study the steady state passive field solutions of resistive MHD. Under two- and $2 \frac{1}{2}$-dimensional symmetries, the planar magnetic field can be written as $\boldsymbol{B}=\hat{\boldsymbol{z}} \times \nabla \psi$, where $\psi$ is the magnetic flux function. Then, the induction equation in (5) with constant resistivity becomes

$$
\frac{\partial \psi}{\partial t}+\boldsymbol{u} \cdot \nabla \psi=\eta \nabla^{2} \psi
$$

We consider the evolution of an initially uniform magnetic field, $\boldsymbol{B}=B_{0} \hat{\boldsymbol{x}}$ or $\psi=B_{0} r e^{i \phi}$, within a vortex. We approximate that the vortex has azimuthal velocity

$$
v_{\phi}= \begin{cases}2 A r, & \text { for } 0<r<r_{0} / 2, \\ 2 A\left(r_{0}-r\right), & \text { for } r_{0} / 2<r<r_{0}, \\ 0, & \text { for } r>r_{0},\end{cases}
$$

and zero radial velocity. $A$ equals the (constant) vorticity within the vortex core. This is roughly the velocity field of the vortex in Figure 4. Then, the evolution of the magnetic field is described by equation (A1) with the boundary conditions $\psi\left(r_{0}\right)=$ $B_{0} r_{0} e^{i \phi}$ and $d \psi\left(r_{0}\right) / d r=B_{0} e^{i \phi}$ at $r=r_{0}$. In a steady state, we can set $\psi=F(r) e^{i n \phi}$ since the coefficients of the equation do not depend explicitly on $\phi$. Note that with the given initial magnetic field and boundary conditions, only the solution with $n=1$ is allowed. Then, the equation for $F$ is given as

$$
\frac{d F}{d r^{2}}+\frac{1}{r} \frac{d F}{d r}-\frac{1}{r^{2}} F-i \frac{Q}{r_{0}^{2}} F=0
$$

where

$$
Q= \begin{cases}q, & \text { for } 0<r<r_{0} / 2 \\ q\left(r_{0} / r-1\right), & \text { for } r_{0} / 2<r<r_{0} \\ 0, & \text { for } r>r_{0}\end{cases}
$$

with $q=2 A r_{0}^{2} / \eta$. Here, $q$ is the magnetic Reynolds number within a factor of 2 .

We solve equation (A3) for $F$ numerically. In Figure 5, we plot the resulting magnetic field lines for the case with $q=10$ (highly diffusive case) and for the case with $q=10^{3}$ (quasi-adiabatic case). From dissipation tests we estimate the magnetic Reynolds number of the vortex in our time-dependent simulations to be larger than $10^{3}$ (see Ryu, Jones, \& Frank 1995; Paper I). Indeed, the fields in Figure $4 b$ resemble those in a vortex simulation with magnetic Reynolds number $10^{3}$ reported in Weiss (1966). The steady state solution we just described allows only for diffusion of magnetic flux, since no change in the field topology is permitted. It allows us to see that such a steady state, diffusive description does not account for flux expulsion from the vortex. If that were the case the magnetic field lines in Figure $4 b$ should have a structure like those in the bottom of Figure 5. Actually, their topologies are fundamentally different in the sense that field in Figure 5 threads completely through the vortex, while it does not in Figure $4 b$ (or any similar figure showing additional field lines for this simulation). The reason is that the field in the time-dependent simulation is subjected to reconnective instabilities that isolate and then destroy magnetic flux in the interior of the vortex.

Balbus, S., \& Hawley, J. 1991, ApJ, 376, 214

Bayly, B. J. 1986, Phys. Rev. Lett., 57, 2160 Bayly, B. J., Orszag, S. A., \& Herbert, T. 1988, Ann. Rev. Fluid Mech., 20,

Biskamp, D. 1993, Nonlinear Magnetohydrodynamics (Cambridge: Cambridge Univ. Press) .1994, Phys. Rep., 237, 179

Biskamp, D., \& Welter, H. 1989, Phys. Fluids B, 1, 1964

Cattaneo, F., \& Vainstein, S. I. 1991, ApJ, 376, L21

\section{REFERENCES}

Chandrasekhar, S. 1961, Hydrodynamic and Hydromagnetic Stability (New York: Oxford Univ. Press)

Corcos, G. M., \& Sherman, F. S. 1984, J. Fluid Mech., 139, 29

Frank, A., Jones, T. W., Ryu, D., \& Gaalaas, J. B. 1996, ApJ, 460, 777 (Paper I)

Harten, A. 1983, J. Comp. Phys., 49, 357

Jun, B. I., Norman, M. L., \& Stone, J. M. 1995, ApJ, 453, 332

Landau, L. D., Lifshitz, E. M., \& Pitaevskii, L. P. 1984, Electrodynamics of Continuous Medium (Oxford: Pergamon) 
Malagoli, A., Bodo, G., \& Rosner, R. 1996, ApJ, 456, 708 (MBR)

Miura, A. 1984, J. Geophys. Res., 89, 801 1990, Geophys. Res. Lett., 17,749

Miura, A., \& Pritchett, P. L. 1982, J. Geophys. Res., 87, 7431

Moffatt, H. K. 1978, Magnetic Field Generation in Electrically Conducting Fluids (Cambridge: Cambridge Univ. Press)

Nordlund, A., et al. 1992, ApJ, 392, 647

Parker, E. N. 1994, Spontaneous Current Sheets in Magnetic Fields (New York: Oxford Univ. Press
Pedelty, J. A., \& Woodward, P. R. 1991, J. Fluid Mech., 225, 101

Pierrehumbert, S. 1986, Phys. Rev. Lett., 57, 2157

Porter, D. H., \& Woodward, P. R. 1994, ApJS, 93, 309

Priest, E. R. 1984, Solar Magnetohydrodynamics (Dordrecht: Reidel)

Ryu, D., \& Jones, T. W. 1995, ApJ, 442, 228

Ryu, D., Jones, T. W., \& Frank, A. 1995, ApJ, 452, 785

Weiss, N. O. 1966, Proc. R. Soc. Lond. A, 293, 310

Zimmer, F., Lesch, H., \& Birk, G. T. 1997, A\&A, in press 